Pacific Journal of Mathematic 


\section{RATIONAL APPROXIMATION ON CERTAIN PLANE SETS}

\section{T. A. MeCullough}

Let $K$ be a compact subset of the complex plane and let $\Omega$ denote its complement. In 1966 Vituskin [11] proved the following generalization of Mergelyan's celebrated theorem on rational approximation [9].

Theorem. (Vituskin). If each boundary point of $K$ is a boundary point of some component of $\Omega$ then $A(K)$, the subset of continuous functions on $K$ which are analytic on the interior of $K$, is the same as $R(K)$, the uniform closure of the rational functions with poles in $\Omega$.

The complexity of Vituskin's techniques justifies the development of alternate approaches to this problem. For a complete discussion of Vituskin's techniques and results see [14]. The alternate approach we have in mind exploits a recent result of Garnett and Glicksberg [5]. Namely, $R(K)=$ $A(K)$ if they have the same representing measures for each point $\varphi \in K$.

We are unable, at present, to prove Vituskin's result. However, if $\Omega_{i}$ denotes the $i^{\text {th }}$ component of $\Omega$, if $A(n, z)$ denotes the annulus $\left\{\left(\frac{1}{2}\right)^{n+1} \leqq|\xi-z| \leqq\left(\frac{1}{2}\right)^{n}\right\}$, and if $\alpha$ denotes analytic capacity, then we prove the following

ThEOREM. If $K$ is such that $(1) \partial(K)$, the boundary of $K$, has finitely many components and (2) $\partial K=\left\{\mathrm{U} \partial \Omega_{i}\right\} \cup\left\{x_{1}\right.$, $\left.x_{2}, \cdots\right\}$, where

$$
\sum_{n=1}^{\infty} 2^{n} \alpha\left(A\left(n, x_{k}\right) \cap \Omega\right)=\infty^{1}
$$

for each $x_{k}$, then $R(K)=A(K)$.

We let $\gamma$ denote logarithmic capacity and we use the associated definitions found in Tsuji [10]. For the definition of analytic capacity and a proof of the fact that $\gamma(E) \geqq \alpha(E)$ see Zalcman [14].

In outline, the proof is as follows. We must show $R(K)$ and $A(K)$ have the same representing measures.

If, for two real measures $\mu_{1}$ and $\mu_{2}$,

$$
\int \ln \left|\frac{1}{z-\xi}\right| d\left(\mu_{1}(\xi)-\mu_{2}(\xi)\right)=0 \quad \text { a.e. (plane Lebesgue measure) }
$$

1 Ahern has recently shown, among other things, (A Condition for Peak Points, to appear) that the hypothesis on the analytic capacity near $x_{k}$ is unnecessary. See addendum. 
then $\mu_{1}=\mu_{2}[10]$. In $\S 2$ we prove a theorem to aid in evaluating the function

$$
P(\mu, z)=\int \ln \left|\frac{1}{z-\xi}\right| d \mu(\xi)
$$

for $z$ in the support of $\mu$, in terms of its values off the support of $\mu$.

The principal result of $\S 3$ is that if conditions (1) and (2) above are satisfied and if $\mu$ is the difference of two representing measures for $R(K)$ and the same $\phi \in K$, then $P(\mu, z)$ is continuous for all $z$ and constant on each component of the boundary of $K$. This last fact allows us to identify the representing measures for $A(K)$ and $R(K)$. This proves the theorem.

The condition (due to Melnikov) on the inner boundary points $x_{i}$ is used only to insure that the points $x_{i}$ are peak points for $R(K)$.

We want to acknowledge observations made by Professor I. Glicksberg (private communication), which (a) simplify our original argument and (b) allow the presence of the exceptional points

$$
\left\{x_{n}\right\} \not \subset\left\{\bigcup \partial \Omega_{i}\right\}
$$

2. A theorem on logarithmic potential for plane measures. Let $E$ be a Borel set in the plane and let $\mu$ be a real measure supported on $E$. Define $P(\mu, z)$, the logarithmic potential of $\mu$, by the formula

$$
P(\mu, z)=\int_{E} \ln \left|\frac{1}{z-\xi}\right| d \mu(\xi) .
$$

$P(\mu, z)$ is obviously harmonic off $E$. We will be concerned with its behavior on $E$ if $\mu$ is a linear combination of representing measures.

The proof of the following theorem structured after Carleson [3]. The use of the equilibrium distribution measures was suggested by Professor P. C. Curtis, Jr.

THEOREM 1. Let $\mu$ be a real measure supported on a compact plane set $E$. Let $z_{0} \in E$ be such that

$$
\int_{E} \ln \left|\frac{1}{z_{0}-\xi}\right| d \mu(\xi)=P\left(\mu, z_{0}\right)
$$

converges absolutely. Let $D\left(r, z_{0}\right)$ be the open disk with radius $r$ and center $z_{0}$. If $V$ is an open set such that

$$
\limsup _{r \rightarrow 0} \frac{\gamma\left(V \cap D\left(r, z_{0}\right)\right)}{r}>0,
$$


then there is a sequence $r_{n} \rightarrow 0$ and probability measures $\nu_{n}$, independent of $\mu$ and supported in $V \cap D\left(r_{n}, r_{0}\right)$, such that

$$
\lim _{n \rightarrow \infty} \int P(\mu, z) d \nu_{n}(z)=P\left(\mu, z_{0}\right) \text {. }
$$

Proof. Suppose $z_{0}=0$. Choose a sequence $r_{n} \rightarrow 0$ so that for some $a>0$

$$
\gamma\left(V \cap D\left(r_{n}, 0\right)\right)>4 a r_{n} .
$$

Now choose compact sets $F_{n} \subset V \cap D\left(r_{n}, 0\right)$ so that

$$
\gamma\left(F_{n}\right)>2 a r_{n} .
$$

Let $\nu_{n}$ be the equilibrium distribution for $F_{n}$. We shall show that $\left\{\nu_{n}\right\}$ is the desired sequence of measures.

First we bound $P\left(\nu_{n}, \xi\right)$. If $|\xi| \geqq 2 r_{n}$ then, since $\nu_{n}$ is positive with total mass one,

$$
\begin{aligned}
\int \ln \left|\frac{1}{z-\xi}\right| d \nu_{n}(z) & =\ln \left|\frac{1}{\xi}\right|+\int \ln \left|\frac{1}{1-z / \xi}\right| d \nu_{n}(z) \\
& \leqq \ln \left|\frac{1}{\xi}\right|+\ln 2 .
\end{aligned}
$$

If $|\xi|<2 r_{n}$ then, by Frostman's theorem [10]

$$
\int \ln \left|\frac{1}{z-\xi}\right| d \nu_{n}(z) \leqq \ln \frac{1}{\gamma\left(F_{n}\right)} \leqq \ln \frac{2}{a}+\ln \left|\frac{1}{\xi}\right| .
$$

Hence $P\left(\nu_{n}, \xi\right) \leqq c+\ln |1 / \xi|$.

Now, for fixed $\rho$,

$$
\left|\int_{|z| \leqq r_{n}} P(\mu, z) d \nu_{n}(z)-\int_{E} \ln \right| \frac{1}{\xi}|d \mu(\xi)|
$$

$$
\begin{aligned}
& \leqq\left|\int_{|\xi|<\rho}\left(\int_{|z| \leqq r_{n}} \ln \left|\frac{1}{z-\xi}\right| d \nu_{n}(z)\right) d \mu(\xi)\right| \\
& +\left|\int_{|\xi| \geqq \rho}\left(\int_{|\xi| \leqq r_{n}}\left(\ln \left|\frac{1}{z-\xi}\right|-\ln \left|\frac{1}{\xi}\right|\right) d \nu_{n}(z)\right) d \mu(\xi)\right| \\
& +\left|\int_{|\xi| \geqq \rho} \ln \right| \frac{1}{\xi}\left|d \mu(\xi)-\int_{E} \ln \right| \frac{1}{\xi}|d \mu(\xi)| .
\end{aligned}
$$

Clearly

$$
\begin{aligned}
& I_{1} \leqq \int_{|\xi|<\rho}\left(c+\ln \left|\frac{1}{\xi}\right|\right) d \mu(\xi) \\
& I_{2} \leqq \int_{|\xi|>\rho} \int_{|z| \leqq r_{n}}\left|\ln \frac{|\xi|}{|z-\xi|}\right| d \nu_{n}(z) d \mu(\xi) \\
& I_{3} \leqq \int_{|\xi|<\rho} \ln \left|\frac{1}{\xi}\right| d \mu(\xi) .
\end{aligned}
$$


Choose $\rho$ so that $I_{1}+I_{3}<\varepsilon / 2$ and then choose $N$ so that

$$
\int_{|z| \leqq r_{N}} \ln \frac{|\xi|}{|z-\xi|} d \nu_{N}(z) \leqq \int_{|z| \leqq r_{N}} \ln \left|\frac{1}{z / \rho-1}\right| d \nu_{N}(z) \leqq \frac{\varepsilon}{2\|\mu\|} .
$$

Then $I_{2} \leqq \varepsilon / 2$. So, for $r_{n} \leqq r_{N}$,

$$
\left|\int_{|z| \leqq r_{n}} P(\mu, z) d \nu_{n}(z)-\int_{E} \ln \right| \frac{1}{\xi}|d \mu(\xi)|<\varepsilon .
$$

To apply Theorem 1 we will need the following estimate.

LEMMA 1. Let $C\left(r, z_{0}\right)$ denote the circle with center $z_{0}$ and radius $r$. Let $V$ be an open set such that $z_{0} \in \partial V$. If for all small $r$ the Lebesgue measure of $\left\{0 \leqq x \leqq r: C\left(x, z_{0}\right) \cap V \neq \varnothing\right\}=r$, then

$$
\limsup _{r \rightarrow 0} \frac{\gamma\left(D\left(r, z_{0}\right) \cap V\right)}{r}>0 \text {. }
$$

Proof. Tsuji [10, Corollary 6, p. 85].

3. The potential generated by representing measures for $R(K)$. Let $\varphi \in K$. Whenever it is convenient we will think of $\varphi$ as a multiplicative linear functional on $R(K)$. A positive measure of mass one supported on $\partial K$ is said to be a representing measure for $R(K)(A(K))$ and the functional (point) $\varphi$ if

$$
f(\phi)=\int_{\partial K} f d \mu \text { for all } f \in R(K)(A(K)) .
$$

We let $M_{\varphi, R}$ denote the collection of all representing measures for $R(K)$ and the point $\varphi$.

There is a distinguished member of $M_{\varphi, R}$ if $\varphi$ is an interior point of $K$. Let $E$ be the component of $K^{0}$, the interior of $K$, which contains $\varphi$. We have in mind the unique measure, $\lambda_{\varphi}$, supported on $\partial E$ with the property that for all $f \in C(K)$ which are harmonic on $K^{0}$

$$
f(\varphi)=\int_{\partial K} f d \lambda_{\varphi} .
$$

We call $\lambda_{\varphi}$ the harmonic measure for $\varphi$. It is not difficult, using hypothesis (2) and the fact that two plane measures with the same logarithmic potential are equal, to see that $\lambda_{\varphi}$ is unique. Also observe that (2) guarantees that $P\left(\lambda_{\varphi}, z\right)$ is continuous for all $z$. To see this, note that each $x \in \partial E$ is a peak point for $R(K)$ and hence is a regular point for $E$. Now use the formula (Tsuji [10], p. 88)

$$
g(z, \varphi)=\ln \left|\frac{1}{z-\varphi}\right|-\int_{\partial E} \ln \left|\frac{1}{z-\xi}\right| d \lambda_{\varphi}(\xi)
$$


and recall that $g(z, \varphi)$ (Green's function) vanishes at regular points.

Let $S_{\varphi, R}$ denote the real linear span of $\left\{\nu-\lambda_{\varphi}: \nu \in M_{\varphi, R}\right\}$. The main result of this section is that hypothesis (2) implies $P(\mu, z)$ is constant on each component of $\partial K$ for each $\mu \in S_{\varphi, R}$. We begin with some technical lemmas.

Lemma 2. If $\phi \in K^{0}$ and $\nu \in M_{\varphi, R}$,

$$
P\left(\mu, z_{0}\right)=\int \ln \left|\frac{1}{z-\xi}\right| d \mu(\xi)
$$

converges absolutely for each $z_{0}$ in the boundary of some component of the complement of $K$.

Proof. Let $\Omega_{i}$ denote a component of $\Omega$ for which $z_{0} \in \partial \Omega_{i}$. If $z_{1}$ and $z_{2}$ belong to $\Omega_{i}$,

$$
\int_{\partial K}\left(\ln \left|\frac{1}{z_{1}-\xi}\right|-\ln \left|\frac{1}{z_{2}-\xi}\right|\right) d\left(\mu-\lambda_{\varphi}\right)=0,
$$

i.e., $P\left(\mu-\lambda_{\varphi}, z\right)$ is constant on $\Omega_{1}$. Let $z_{n} \in \Omega_{i}$ and $z_{n} \rightarrow z_{0} \in \partial \Omega_{i}$. If $\delta$ is the diameter of $K$ then we may assume

$$
\ln \frac{1}{3 \delta}<P\left(\mu, z_{n}\right)=P\left(\mu-\lambda_{\varphi}, z_{n}\right)+P\left(\lambda_{\varphi}, z_{n}\right) .
$$

Now $P\left(\mu-\lambda_{\varphi}, z_{n}\right)=C$ and

$$
\left|P\left(\lambda_{\varphi}, z_{n}\right)\right|=|\ln | \frac{1}{\varphi-z_{n}}|| \leqq M
$$

imply

$$
\liminf _{z_{n} \rightarrow z_{0}} P\left(\mu, z_{n}\right)<\infty .
$$

By the lower continuity,

$$
P\left(\mu, z_{0}\right) \leqq \liminf _{z \rightarrow z_{0}} P(\mu, z) \leqq C+M,
$$

and the lemma is proved.

Lemma 3. Fix a $\varphi \in K^{0}$ and $a \nu \in M_{\varphi, R}$. For each $z \in \mathbf{U} \partial \Omega_{i}$, where $\Omega_{i}$ is a component of $\Omega$, let the set $W(z)$ be the union of all connected subsets of $\bar{\Omega}$ containing $z$ on which $P\left(\nu-\lambda_{\varphi}, z\right)$ is a constant. We assert that

$$
P(\nu, t)=\int \ln \left|\frac{1}{t-\xi}\right| d v(\xi)
$$


converges absolutely for $t \in \overline{W(z)}$.

Proof. We need only consider $t \in \partial W(z)$. For such $t$ use the proof of Lemma 2 (beginning with line 4) with $\Omega_{i}$ replaced by $W(z)$.

Lemma 4. For $\varphi \in K^{0}$ and $\mu \in S_{\varphi, R}, P(\mu, z)$ is constant on $\bar{\Omega}_{i}$ for each component $\Omega_{j}$ of $\Omega$.

Proof. By definition $\mu=\Sigma \alpha_{i} \mu_{i}$, where the summation is finite, $\mu_{i}=\nu_{i}-\lambda_{\varphi}$, and $\nu_{i} \in M_{\varphi, R}$. Then

$$
P(\mu, z)=\Sigma \alpha_{i} P\left(\mu_{i}, z\right)=\Sigma \alpha_{i} P\left(\nu_{i}-\lambda_{\varphi}, z\right)
$$

and

$$
\left.P\left(\nu_{i}-\lambda_{\varphi}, z\right)\right|_{\Omega_{j}}=C_{i j}
$$

By Lemma 2, $P\left(\nu_{i}-\lambda_{\varphi}, z\right)$ converges absolutely for each $z \in \partial Q_{j}$. Taking $\Omega_{j}$ to be the open set in the hypothesis of Lemma 1, we conclude from Theorem 1 and Lemma 1 that for $z \in \partial \Omega_{j}$,

$$
C_{i j}=P\left(\nu_{i}-\lambda_{\varphi}, z\right)=P\left(\mu_{i}, z\right) .
$$

Thus $P(\mu, z)=\Sigma \alpha_{i} C_{i j}$ is a constant on $\bar{\Omega}_{j}$.

THEOREm 2. If $\partial K$ satisfies (2) and $\varphi \in K^{0}$ then, for each $\nu \in M_{\varphi, R}$, $P\left(\nu-\lambda_{\varphi}, z\right)$ is constant on each component of $\partial K$.

Proof. Let $W(z)$ be as in Lemma 3. If $x_{n} \in \overline{W(z)}$ for some $z \in \bigcup \partial \Omega_{i}$, then by Lemma $3, P\left(\nu-\lambda_{\varphi}, x_{n}\right)$ converges absolutely. If $x_{n} \notin \bigcup\left\{\overline{W(z)}: z \in \partial \Omega_{i}\right\}$, then set $W\left(x_{n}\right)=\left\{x_{n}\right\}$.

Assert that each $W(z)$ is a closed set. To prove this we verify the hypothesis of Lemma 1 so that we may use Theorem 1. Fix $z \in \bigcup \partial \Omega_{i}$, let $z_{1} \in \partial W(z)$, and pick $r_{1}>0$ so that $C\left(r, z_{1}\right) \cap W(z) \neq \varnothing$ for all $0<r \leqq r_{1}$ (recall that $W(z)$ is connected). Let

$$
E=\left\{0<r \leqq r_{1}: C\left(r, z_{1}\right) \cap \Omega \cap W(z)=\varnothing\right\} \cup\{0\} .
$$

Evidently the complement of $E$ is open. We assert that $E$ is countable. First observe that for each component $\Omega_{i}$ of $\Omega$ there can be at most two distinct $r \in E$ with $C(r, z) \cap \bar{\Omega}_{i} \neq \varnothing$. Now if $r \in E$ there is a $y \in C\left(r, z_{1}\right) \cap W(z) \cap \bar{\Omega}$ and either $y=x_{n}$, for some $n$, or $y \in \partial \Omega_{i}$ for some $i$. Hence $E$ is countable. Since $E$ is closed and countable, we have, for small $r$, the Lebesgue measure of

$$
\left\{x \leqq r ; C\left(x, z_{1}\right) \cap W(z) \cap \Omega \neq \varnothing\right\}=r .
$$

By Lemma 1 


$$
\limsup _{r \rightarrow 0} \frac{\gamma\left(W(z) \cap D\left(r, z_{1}\right) \cap \Omega\right)}{r} \geqq c>0 .
$$

By Theorem 1, with $V=W(z) \cap \Omega$, we have

$$
P\left(\nu-\lambda_{\varphi}, z_{1}\right)=P\left(\nu-\lambda_{\varphi}, z\right)
$$

and hence $W(z)$ is closed.

Finally note that, by Lemma 4 there are only countably many distinct sets $W(z)$ for $z \in \bigcup \partial \Omega_{i} \bigcup\left\{x_{1}, x_{2}, \cdots\right\}$.

Let $\Gamma$ be a component of $\partial K$. If $\Gamma \not \subset W(z)$ for some $z$, then a countable union of the $W(z)$ cover $\Gamma$. However it is standard fact [8] that a connected set cannot be the disjoint union of countably may closed sets. Hence $\Gamma \subset W(z)$ for some $z \in \bigcup \partial \Omega_{i} \bigcup\left\{x_{1}, x_{2}, \cdots\right\}$ (indeed for some $z \in \bigcup \partial \Omega_{i}$, if $\partial K$ contains no singletons) and $P\left(\nu-\lambda_{\varphi}, z\right)$ is constant on $\Gamma$.

CoRollary. If, in addition to the above hypothesis, $\partial K$ has a finite number of components then, for $\mu \in S_{\varphi, R}, P(\mu, z)$ is a continuous function of $z$ and is harmonic except on $\partial K$.

Proof. Write $P(\mu, z)=\sum \alpha_{i} P\left(\mu_{i}, z\right)$ where $\mu_{i}+\lambda_{\varphi}=\nu_{i} \in M_{\varphi, R}$. Thus $\left.P\left(\nu_{i}, z\right)\right|_{\partial K}$ is continuous. Hence, by Tsuji III. 2. [10], $P\left(\mu_{i}, z\right)=$ $P\left(\nu_{i}, z\right)-P\left(\lambda_{\varphi}, z\right)$ is continuous for all $z$.

4. Representing measures for $R(K)$ and $A(K) . A(K)$ is the Banach algebra of all functions on $K$ and analytic on $K^{0}$. Arens [2] shows that multiplicative linear functionals on $A(K)$ can be identified with the points of $K$, so that $A(K)$ and $R(K)$ have the same maximal ideal space. In this section we show that $R(K)$ and $A(K)$ have the same representing measures for each $\varphi \in K$ provided that hypothesis (1) and (2) hold.

As Glicksberg observed, it is sufficient to show that for each $\varphi \in K$ any $\mu \in S_{\varphi, R}$ annihilates $A(K)$. For if $\nu \in M_{\varphi, R}$ then $\nu-\lambda_{\varphi} \in S_{\varphi, R}$ so that $\nu$ is a representing measure for $A(K)$. Hence by Garnett and Glicksberg [5] we are done. Finally note (i) by Silov's Idempotent theorem we can assume $K$ is connected and then (ii) there are no isolated points in $\partial K$ since $K$ is compact.

LEMMA 5. If $\partial K$ has $n+1$ components and $\varphi \in K^{0}$ then dimension of $S_{\varphi, R} \leqq n$.

Proof. First suppose $\nu_{1}, \cdots, \nu_{n+2} \in S_{\varphi, R}$. For each $\nu_{j}$, let $C_{j k}=$ $\left.P\left(\nu_{j}, z\right)\right|_{\Gamma_{k}}$, where $\Gamma_{k}$ is the $k^{\text {th }}$ component of $\partial K$. By Theorem 2 the $C_{j k}$ 's are constant. The matrix $\left(C_{j k}\right)$ is obviously singular and hence 
there are real scalars $\alpha_{1}, \cdots, \alpha_{n+2}$ such that

$$
\left.\Sigma \alpha_{j} P\left(\nu_{j}, z\right)\right|_{\partial K} \equiv 0 \quad j \in\{1, \cdots, n+2\} .
$$

However, by the corollary to Theorem 2 the potential generated by the measure

$$
\Sigma \alpha_{j} \nu_{j} \in S_{\varphi, R}
$$

is a continuous function and is harmonic except on $\partial K$ where, by $(*)$, it is zero. Hence by the maximum principle for harmonic functions

$$
P\left(\Sigma \alpha_{j} \nu_{j}, z\right)=0 \quad \text { all } z \text {. }
$$

Since the zero measure is the only measure with zero potential we conclude that the dimension of $S_{\varphi, R} \leqq n+1$.

Finally, if $\Omega_{\infty}$ is the unbounded component of $\Omega$ then $P(\nu, z)=0$ on $\bar{\Omega}_{\infty}$ for all $\nu \in S_{\varphi, R}$. Hence dimension of $S_{\varphi, R} \leqq n$.

Lemma 6. If $K$ satisfies (1) and (2) and $\varphi \in K^{0}$ then $S_{\varphi, R}$ annihilates $A(K)$.

Proof. Essentially the proof is the identification of a basis for $S_{\varphi, A}$. We construct measures $\mu_{i}$ on $\partial K$ as suggested by Ahern and Sarason [1] (see also Garnet and Glicksberg [5]).

The hypothesis on $K$ implies $\bar{\Omega}$ has a finite number of components. Each component, $I_{i}^{*}$, of $\bar{\Omega}$ may separated from the other components by a finite number of simple smooth oriented contours whose union we denote by $\Lambda_{i}$. For $f \in C(\partial K)$, let $\widetilde{f}$ be its harmonic extension to $K^{0}$ and for each $\Gamma_{i}^{*}$, except the one containing $\infty$, let

$$
\int_{\partial K} f d \mu_{i}=\frac{1}{2 \pi} \int_{\Lambda_{i}} \frac{\partial}{\partial n} \tilde{f} d s \text {. }
$$

$\left(\partial / \partial n\right.$ is the normal derivative). The following facts about $\mu_{i}$ are easily established:

(1) if $f \in A(K), \int_{\partial K} f d \mu_{i}=0$

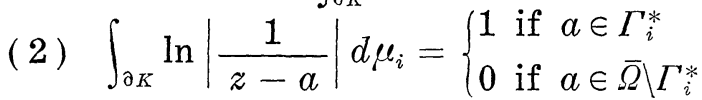

By Theorem 2, for $\nu \in S_{\phi, R}, P(\nu, z)$ is constant on each component $\Gamma_{i}^{*}$ of $\bar{\Omega}$ hence, for all $z$,

$$
P(\nu, z)=\Sigma \alpha_{i} P\left(\mu_{i}, z\right) \quad i=1, \cdots, n-1 .
$$

Thus $\nu=\Sigma \alpha_{i} \mu_{i}$, i.e., $\nu \perp A(K)$.

CoRollary. $R(K)$ and $A(K)$ have the same representing measures. 
Proof. Now we need only concern ourselves with points $z \in \partial K$. If $z \in \bigcup \partial \Omega_{i}$ then it is easy to see that

$$
\Sigma 2^{n} \alpha(A(n, z) \cap \Omega)=\infty .
$$

If $z \in \partial K-\bigcup \partial \Omega_{i}$ then, by assumption,

$$
\Sigma 2^{n} \alpha(A(n, z) \cap \Omega)=\infty .
$$

In either case by [4, Th. 3.5], $z$ is a peak point for $R(K)$ so that the only representing measure is the unit mass at $z$. Hence $A(K)$ and $R(K)$ have the same representing measures for each $z \in K$.

The desired generalization of Mergelyan's theorem now follows from Garnett and Glicksberg [5, Th. 1.7].

5. Added August 19, 1968. Since this paper was written Ahern (A condition for Peak Points, to appear in the Duke Math. Journal) has proven, among other things, that each $x_{n} \in \partial K-\left\{\bigcup \partial \Omega_{i}\right\}$ is a peak point provided that $\partial K-\left\{\mathbf{U} \partial \Omega_{i}\right\}$ is countable. Ahern's argument can be simplified as follows. First, as Ahern observes, because $\partial K$ has finitely many components each $x_{n}$ is a regular point for $K$, we can apply Theorem 2. Suppose $x_{n}$ is not a peak point. By Wilkin's theorem, the part, $P$, containing $x_{n}$ has positive planar measure. Since $P \cap\left(\bigcup \partial \Omega_{i}\right)=\phi, P$ contains a point $\phi \in K^{0}$. Let $\mu \in M_{x_{n}, R}, \mu\left(\left\{x_{n}\right\}\right)=0$. By a theorem of Bishop there exists $0<c<1$ and $\mu_{\phi} \in M_{\phi, R}$ such that $\mu_{\phi}-c \mu \geqq 0$. Hence $\nu_{\dot{\phi}}=\left(\mu_{\dot{\phi}}-c \mu\right)+c \delta_{x_{n}} \in M_{\dot{\phi}, R}$ and $P\left(\nu_{\dot{\phi}}, x_{n}\right)=\infty$. This contradicts Theorem 2. (An argument along these lines was suggested to me independently by A. M. Davie and J. Garnett.)

\section{REFERENCES}

1. P. R. Ahern and D. Sarason, The $H^{p}$ spaces of a class of function algebras, Acta Math. 117 (1967), 123-163.

2. R. Arens, The maximal ideals of certain function algebras, Pacific J. Math. 8 (1958), 641-648.

3. L. Carleson, Mergelyan's theorem on uniform polynomial approximation, Math. Scand. 15 (1964), 167-175.

4. P. C. Curtis, Jr., Peak points for algebras of analytic functions (to appear)

5. J. Garnett and I. Glicksberg, Algebras with the same multiplicative measures (to appear)

6. I. Gliksberg, Dominant representing measures and rational approximation (to appear)

7. I. Glicksberg and J. Wermer, Measures orthogonal to Dirichlet algebras, Duke Math.

J. 30 (1963), 661-666.

8. F. Hausdorff, Set Theory, Chelsea Pub. Co., New York, 1962.

9. S. N. Mergelyan, Uniform approximation to functions of a complex variable, Amer. Math. Soc. Transl., No. 101.

10. M. Tsuji, Potential theory in modern function theory, Maruzen Co., Ltd., Tokyo, 1959. 
11. A. G. Vituskin, Necessary and sufficient conditions on a set in order that any continuous function analytic at the interior points of the set may admit of uniform approximation by rational functions, Soviet Math. Pokl. 7 (1966), 1622-1625.

12. J. J. Walsh, Uber die Entricklung einer harmonischen Function nach haromischen, Polynomen, J. Reine Adgew. Math. 159 (1928), 197-209.

13. D. Wilken, Lesbesgue measure for parts of $R(X)$, Proc. Amer. Math. Soc. 18 (1967), 508-512.

14. L. Zalcman, Analytic capacity and rational approximation, Multilithed notes. (To be published in Springer Math Notes.)

Received February 26, 1968. This paper is based on the author's doctoral dissertation written at the University of California at Los Angeles under the supervision of Professor P. C. Curtis, Jr. Improvements, as noted, were suggested by Professor I. Glicksberg, University of Washington. The preparation of this paper was sponsored in part by NSF Grant GP-5138.

UNiversity of CALIFornia, Los ANGELES, AND

California State College, Long Beach 


\section{PACIFIC JOURNAL OF MATHEMATICS}

\section{EDITORS}

H. ROYDEN

Stanford University

Stanford, California

\author{
R. R. Phelps \\ University of Washington \\ Seattle, Washington 98105
}

J. DugundJI

Department of Mathematics

University of Southern California

Los Angeles, California 90007

RICHARD ARENS

University of California

Los Angeles, California 90024

\section{ASSOCIATE EDITORS}

E. F. BECKenbaCH

B. H. NEUMANN

F. WOLF

K. YoSHIDA

\section{SUPPORTING INSTITUTIONS}

\author{
UNIVERSITY OF BRITISH COLUMBIA \\ CALIFORNIA INSTITUTE OF TECHNOLOGY \\ UNIVERSITY OF CALIFORNIA \\ MONTANA STATE UNIVERSITY \\ UNIVERSITY OF NEVADA \\ NEW MEXICO STATE UNIVERSITY \\ OREGON STATE UNIVERSITY \\ UNIVERSITY OF OREGON \\ OSAKA UNIVERSITY \\ UNIVERSITY OF SOUTHERN CALIFORNIA
}

\author{
STANFORD UNIVERSITY \\ UNIVERSITY OF TOKYO \\ UNIVERSITY OF UTAH \\ WASHINGTON STATE UNIVERSITY \\ UNIVERSITY OF WASHINGTON \\ $\stackrel{*}{*} \stackrel{*}{*} \stackrel{*}{*}$ AMERICAN MATHEMATICAL SOCIETY \\ CHEVRON RESEARCH CORPORATION \\ TRW SYSTEMS \\ NAVAL WEAPONS CENTER
}

The Supporting Institutions listed above contribute to the cost of publication of this Journal, but they are not owners or publishers and have no responsibility for its content or policies.

Mathematical papers intended for publication in the Pacific Journal of Mathematics should be in typed form or offset-reproduced, double spaced with large margins. Underline Greek letters in red, German in green, and script in blue. The first paragraph or two must be capable of being used separately as a synopsis of the entire paper. It should not contain references to the bibliography. Manuscripts, in duplicate if possible, may be sent to any one of the four editors. Please classify according to the scheme of Math. Rev. 36, 1539-1546. All other communications to the editors should be addressed to the managing editor, Richard Arens, University of California, Los Angeles, California, 90024.

50 reprints are provided free for each article; additional copies may be obtained at cost in multiples of 50 .

The Pacific Journal of Mathematics is published monthly. Effective with Volume 16 the price per volume (3 numbers) is $\$ 8.00$; single issues, $\$ 3.00$. Special price for current issues to individual faculty members of supporting institutions and to individual members of the American Mathematical Society: $\$ 4.00$ per volume; single issues $\$ 1.50$. Back numbers are available.

Subscriptions, orders for back numbers, and changes of address should be sent to Pacific Journal of Mathematics, 103 Highland Boulevard, Berkeley, California, 94708.

PUBLISHED BY PACIFIC JOURNAL OF MATHEMATICS, A NON-PROFIT CORPORATION

Printed at Kokusai Bunken Insatsusha (International Academic Printing Co., Ltd.), 7-17, Fujimi 2-chome, Chiyoda-ku, Tokyo, Japan. 


\section{Pacific Journal of Mathematics \\ Vol. 29, No. $3 \quad$ July, 1969}

Herbert James Alexander, Extending bounded holomorphic functions from certain subvarieties of a polydisc ...................... 485

Edward T. Cline, On an embedding property of generalized Carter

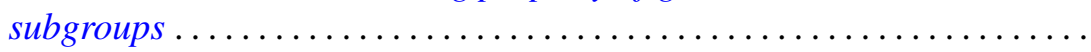

Roger Cuppens, On the decomposition of infinitely divisible characteristic functions with continuous Poisson spectrum. II ...............

William Richard Emerson, Translation kernels on discrete Abelian

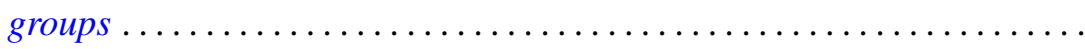

Robert William Gilmer, Jr., Power series rings over a Krull domain ....... 543

Julien O. Hennefeld, The Arens products and an imbedding theorem ...... 551

James Secord Howland, Embedded eigenvalues and virtual poles ........ 565

Bruce Ansgar Jensen, Infinite semigroups whose non-trivial homomorphs are all isomorphic .............................. 583

Michael Joseph Kascic, Jr., Polynomials in linear relations. II .......... 593

J. Gopala Krishna, Maximum term of a power series in one and several

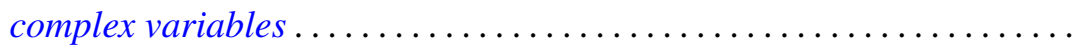

Renu Chakravarti Laskar, Eigenvalues of the adjacency matrix of cubic lattice graphs ...................................

Thomas Anthony Mc Cullough, Rational approximation on certain plane

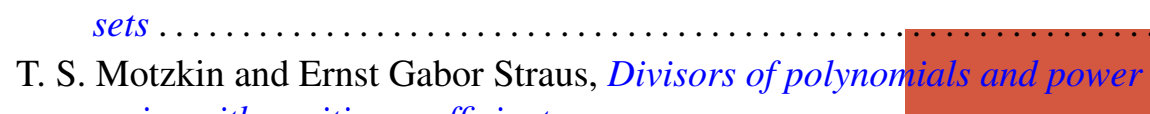
series with positive coefficients .

Graciano de Oliveira, Matrices with prescribed characteristic polynomial and a prescribed submatrix.

Graciano de Oliveira, Matrices with prescribed characteristic polynomial and a prescribed submatrix. II .

Donald Steven Passman, Exceptional 3/2-transitive permutation groups .................................

Grigorios Tsagas, A special deformation of the metric with no negative sectional curvature of a Riemannian space............

Joseph Zaks, Trivially extending decompositions of $E^{n}$ 